\title{
Fibre Optic Readout of Microcantilever Arrays for Fast Microorganism Growth Detection
}

\author{
N. Maloney, ${ }^{1}$ G. Lukacs, ${ }^{1}$ N. Nugaeva, ${ }^{2}$ W. Grange, ${ }^{3}$ J. P. Ramseyer, ${ }^{4}$ J. Jensen, ${ }^{1}$ and M. Hegner ${ }^{1}$ \\ ${ }^{1}$ The School of Physics and The Centre for Research on Adaptive Nanostructures and Nanodevices (CRANN), Trinity College Dublin, \\ Dublin 2, Ireland \\ ${ }^{2}$ Toronto Western Hospital, UHN, 399 Bathurst Street, Toronto, ON M5T 2S8, Canada M5T 2S8 \\ ${ }^{3}$ Institut Jacques Monod, CNRS-Université Paris Diderot, 75205 Paris Cedex 13, France \\ ${ }^{4}$ Department of Chemistry, University of Basel, Klingelbergstrasse 80, 4056 Basel, Switzerland
}

Correspondence should be addressed to N. Maloney, maloneng@tcd.ie

Received 14 June 2011; Accepted 6 July 2011

Academic Editor: Maria Tenje

Copyright ( $) 2012$ N. Maloney et al. This is an open access article distributed under the Creative Commons Attribution License, which permits unrestricted use, distribution, and reproduction in any medium, provided the original work is properly cited.

\begin{abstract}
We present a fibre-optic-based device for the automated readout of microcantilever arrays for fast microorganism growth detection. We determined the ability of our device to track shifts in resonance frequency due to an increase in mass on the cantilever surface or changes in mechanical stiffness. The resonance frequency response of $7 \mu \mathrm{m}$ thick agarose-functionalised cantilevers was tracked as humidity levels were varied revealing a mass responsivity of $\sim 51 \pm 1 \mathrm{pg} / \mathrm{Hz}$. The resonance response of microcantilevers coated with Aspergillus niger (A. niger) spores was monitored for $>48 \mathrm{~h}$ revealing a growth detection time of $>4 \mathrm{~h}$. The growth of mycelium along the cantilevers surface is seen to result in an increase in resonance frequency due to the reinforcement of the cantilever structure. The use of our fibre optic detection technique allows data to be recorded continuously and faster than previously reported.
\end{abstract}

\section{Introduction}

Microbial infection through contamination still poses a threat in areas such as the pharmaceutical, cosmetic, and food industries. For this reason, quality control tests are performed to ensure that microbial contamination does not occur and that preservatives are functional. The survival of a microbial population is dependent on the provision of suitable nutrition and environmental conditions (relative humidity $>93 \%$, temperature $=25^{\circ} \mathrm{C}-37^{\circ} \mathrm{C}$ ). Conventional methods to date rely on contaminating a sample containing preservatives with prescribed inocula of microorganism on a petri dish, followed by counting the colonies present. An example of this would be a preservative efficacy test for a pharmaceutical product [1] where, under the effect of the preservative living bacteria should show a log reduction of 3 after $24 \mathrm{~h}$ and fungi should show a $\log$ reduction of 2 after 7 days. These inexpensive manual methods provide the desired sensitivity and growth information but are time consuming and require highly skilled personnel to perform them. For this reason, there is a need for the development of automated, highly sensitive, label-free and real-time detection systems.

The reported use of microcantilever resonators as biosensors has risen in recent years. Typically, detection is based on either (i) tracking the deflection caused by induced surface stress changes on the cantilever surface by adsorption of target biomolecules (static mode) $[2,3]$ or (ii) tracking the resonance frequency changes caused by mass adsorption on the cantilever surface (dynamic mode). Using static mode, single base pair mismatches have been detected in DNA hybridization experiments [4] while the detection of Escherichia coli (E. coli) has also been reported using an antibody-immobilized microcantilever with a detection limit of $1 \times 10^{6} \mathrm{cfu} / \mathrm{mL}$ [5]. Using dynamic mode, a mass resolution of 1 attogram $\left(10^{-18} \mathrm{~g}\right)$ has been achieved [6], while the detection of bacterial cells [7], virus particles [8,9], and biomolecules [10] has also been described. The use of microcantilever arrays for the detection of viable microorganism growth has previously been reported in [11-14]. These measurements, based on the optical beam deflection method 
[15], were performed using a scanning probe MultiMode head operated in a controlled environment. Manual manipulation of the laser spot was required for the readout of several cantilevers. This paper presents the development and successful application of an automatised fibre-optic-based readout technique of cantilever arrays for microorganism growth detection. The principle of operation relies on the modulation of the optical signal, by an oscillating cantilever, carried by the optic fibres to a photodiode detector. The mechanism of detection is similar to that used in [11, 12] where the absorption of water on the functionalised cantilever surface due to microorganism growth results in a resonance frequency shift.

\section{Materials and Methods}

2.1. Chemicals and Reagents. All chemicals and reagents are of analytical grade and were purchased from Sigma-Aldrich (Arklow, Ireland), unless otherwise stated.

2.2. Aspergillus niger Culture Method. Aspergillus niger (A. niger, CIP 1431.83) spores were obtained from Institut Pasteur (Paris, France). Spores were cultured on potato dextrose agar (PDA) (Oxoid, Basel, Switzerland) slants at $35^{\circ} \mathrm{C}$ for five days and then stored at $4^{\circ} \mathrm{C}$ until required. Spores were suspended in $4 \mathrm{~mL}$ of malt extract broth (ME). Spores were harvested by centrifugation (10,000 rpm, $5 \mathrm{~min}$ ) (Genofuge 16M, Techne) of $3 \mathrm{~mL}$ of this suspension. The resulting pellet was resuspended in a $200 \mu \mathrm{L}$ solution of $\mathrm{ME}$ and deionised water $(1: 10)$. These steps take an average of 20 mins to complete after which the spore suspension is used for cantilever functionalisation (Section 2.3).

\subsection{Cantilever Array Preparation and Functionalisation.} Cantilever arrays used were obtained from the microfabrication group located in the IBM Zurich Research Laboratory. Each array consists of eight cantilevers separated by a pitch of $250 \mu \mathrm{m}$ with length, width, and thickness dimensions of $500 \mu \mathrm{m}, 100 \mu \mathrm{m}$, and $7 \mu \mathrm{m}$, respectively. Two types of array (array A and array B) were prepared. Array type A were cleaned for 10 min in Piranha solution $\left(\mathrm{H}_{2} \mathrm{SO}_{4}: \mathrm{H}_{2} \mathrm{O}_{2} ; 1: 1\right)$ and subsequently washed in a solution of nanopure water and ethanol $(1: 1)$ and then nanopure water. This procedure was repeated twice, and the array was then washed using HPLC grade isopropanol. Array type B was cleaned by exposing it to 0.3 mbar $\mathrm{O}_{2}$ plasma for $3 \mathrm{~min}$ (PICO Barrel Asher; Diener electronic GmbH + Co. KG, Nagolderstrasse 61, D72224 Ebhausen, Germany). After cleaning, a self-assembled monolayer was formed on the arrays by immersion in a silane solution (3-glycidyloxypropyl-trimethoxysilane: $\mathrm{N}$ ethyldiisopropylamine: water free toluene; $1: 1: 100)$ for $45 \mathrm{~min}$ and then washed twice in water-free toluene for $15 \mathrm{~min}$. The array was dried using nitrogen gas and then stored under vacuum. This step provides an epoxy-activated surface which is reactive, in alkali conditions, towards primary hydroxyl groups (e.g., agarose).

Seakem Gold Agarose (Bioconcept; NH, USA) was dissolved in nanopure water at a concentration of $1 \%$

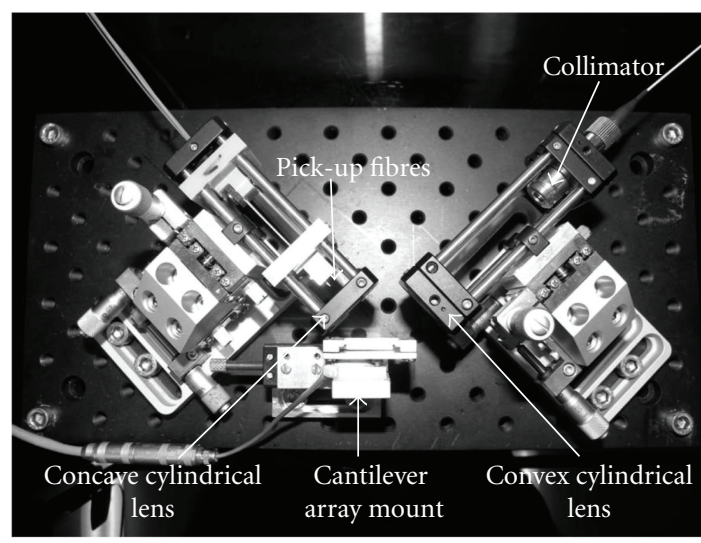

FIgURE 1: Photograph of fibre optic cantilever device. A collimated laser beam is focused into a line along a cantilever array using a convex cylindrical lens. The deflected signal from each cantilever is directed into one of eight optic fibres (one for each cantilever) using a concave cylindrical lens. The optical axis is maintained using two cage systems, which are mounted on two gothic arch XYZ translation stages. Horizontal distance from hole to hole is $25 \mathrm{~mm}$ on breadboard.

(wt/vol) and stored at $4^{\circ} \mathrm{C}$. Prior to use the agarose gel solution was melted. The $\mathrm{pH}$ of the agarose solution was adjusted to $\sim 11.9$ by adding $2 \mathrm{M} \mathrm{NaOH}$. Glass capillaries (King Precision Glass, Inc.; Calif, USA) were used to agarose functionalise the cantilever arrays as described in [16]. In order to prevent gelatinisation of the agarose solution in the capillary tubes, they were preheated using a radiation lamp (Osram; 8401 Winterthur, Switzerland). In the case of array type A, cantilevers were exposed to the agarose solution for 1 second. In the case of array type B, cantilevers were exposed to the agarose solution for 1,5 , or 10 seconds to obtain a different agarose thickness on each cantilever. The nutritional soaking of the agarose layer and deposition of A. niger spores on the cantilever were performed in parallel using glass capillaries as previously mentioned. The spore suspension used was prepared as discussed in Section 2.2. Cantilevers on array type A were immersed in the spore suspension for $10 \mathrm{~min}$. The in situ reference cantilever was immersed in 10\% ME broth without spores for $10 \mathrm{~min}$. The array was immediately placed inside the test chamber at $94 \%$ $\mathrm{RH}$ and $30^{\circ} \mathrm{C}$.

2.4. Apparatus and Measurement. All components were purchased from Thorlabs (Cambridgeshire CB7 4EX, UK), unless otherwise stated.v Experiments were performed using a custom built device (Figure 1) based on the optical beam deflection method. The deflected optical beam is captured using optic fibres. When actuated at its resonance frequency, the motion of a cantilever results in a modulation in the intensity of the optical beam captured by the optic fibres. By recording the intensity modulation, the resonance frequency of a cantilever can be determined and tracked. The optical axis of the device is maintained using $16 \mathrm{~mm}$ cage plates (SP03) and cage rods (SR3) which are mounted on two gothic-arch XYZ translation stages (9061 XYZ, Newport, 


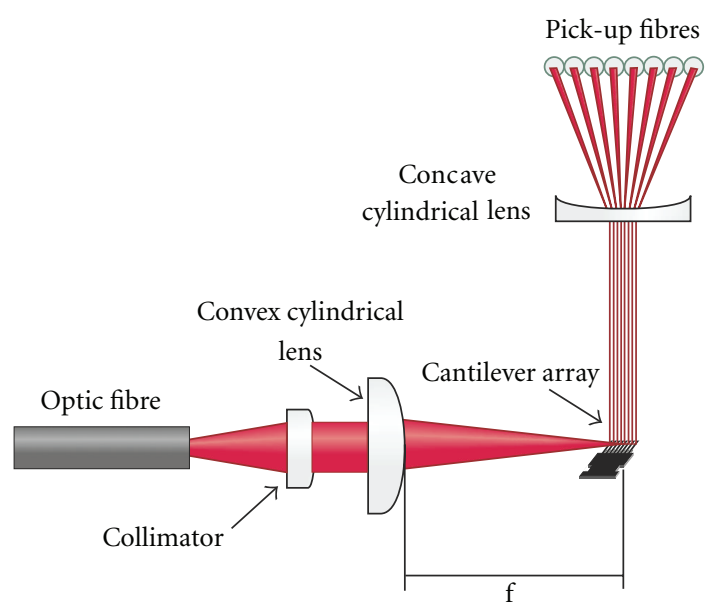

FIGURE 2: Illustration outlining optics of fibre optic cantilever device. Description is given in Section 2.4.

Calif, USA). A collimated laser beam (beam diameter $=3.4 \mathrm{~mm}$ ) is provided to the system using a pig-tailed laser diode (LPS-830-FC, $\lambda=830 \mathrm{~nm}$, maximum power $12 \mathrm{~mW}$ ) which is accompanied by a collimator (F280FC$\mathrm{B})$. The laser diode is driven with an external module (ITC502-Benchtop Laser Diode and Temperature Controller $\pm 200 \mathrm{~mA} / 16 \mathrm{~W}$ ) and is temperature controlled (TCLDM9TE-Cooled Mount). The collimated beam is focused into a line, with a width of $\sim 6 \mu \mathrm{m}$, along a cantilever array using a convex cylindrical lens (47764, $f=20 \mathrm{~mm}$; Edmund Optics, $\mathrm{UK})$. The reflection from each cantilever is directed into eight pick-up fibres (BFL48-200, core diameter $=0.2 \mathrm{~mm}$ ), one corresponding to each cantilever in the array, via a concave cylindrical lens (LK1363L1-B, $f=-7.7 \mathrm{~mm}$ ). Figure 2 shows an illustration of this setup. The pick-up fibres are coupled to a custom built photodetector device where a voltage is produced proportional to the intensity of the detected light. The voltage generated is amplified using a low-noise voltage preamplifier (SR560, Stanford Research Systems, Calif, USA). Actuation of the cantilever array is performed using a custom made stage containing a $15 \mathrm{~mm}$ $\times 15 \mathrm{~mm} \times 1 \mathrm{~mm}$ piezo ceramic plate (EBL\#2, EBL Products Inc.; Conn, USA).

LabVIEW (National Instruments, Tex, USA) is used to control our instrument and to measure the resonance frequencies of cantilevers in an array in a time multiplexed fashion. Three boards, NI PCI-5412 (100 MS/s, 14-Bit Arbitrary Waveform Generator; National Instruments, Tex, USA), NI PCI-6010 (16-Bit, $200 \mathrm{kS} / \mathrm{s}, 16$ Analog Input Multifunction DAQ; National Instruments, Tex, USA), and NI 5112 (High speed digitiser; National Instruments, Tex, USA), allow us (i) to output voltages on the before-mentioned piezo plate, (ii) to acquire and process voltages from the custom built photodetector device, and (iii) to control the current applied to the pigtailed laser diode.

In order to obtain suitable conditions for microorganism growth, a custom environmental chamber (5500-8139 A; Electro Tech Systems Inc., Pa, USA) is used to regulate temperature and humidity. Temperature is generated using

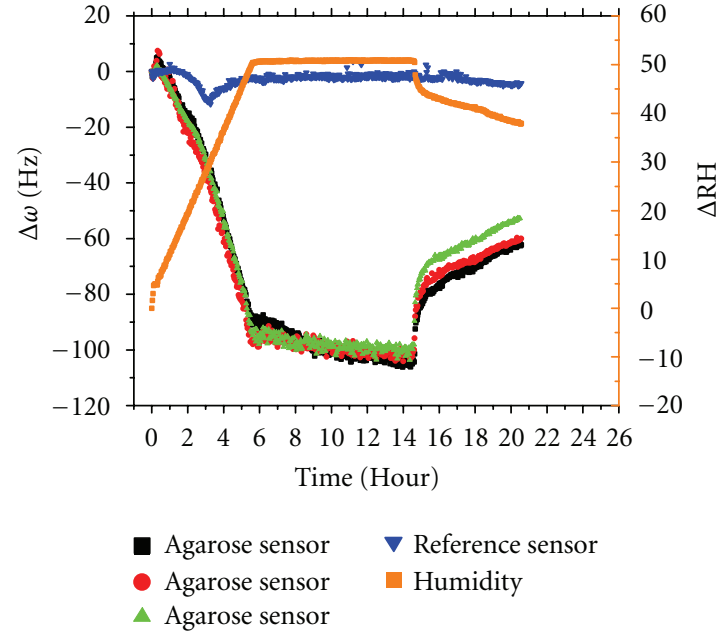

FIGURE 3: Resonance frequency response of agarose functionalised cantilevers. Agarose functionalized cantilevers show an increased response to changes in humidity. Zero point on right hand axis corresponds to $44 \% \mathrm{RH}$.

a heating element housed inside the chamber. Humid air is pumped into the chamber using an ultrasonic humidification system (5472-3; Electro Tech Systems Inc.; PA 19038 USA). Humidity and temperature are regulated using a temperature-compensated humidity sensor (554; Electro Tech Systems Inc, Pa, USA) in conjunction with a microprocessor controller (5200 441-431; Electro Tech Systems Inc., $\mathrm{Pa}$, USA).

\section{Results and Discussion}

The detection of E. coli and A. niger growth using cantilever arrays has been reported in [11-14]. However, as mentioned previously, this work was performed manually using a scanning probe MultiMode head with NanoScope control software (Digital Instruments, Santa Barbara, Calif, USA). For our fibre optic device, the following tests were performed to ensure that continuous resonance frequency changes, due to increasing mass on the cantilever surface, can be detected.

\subsection{Response of Agarose-Functionalised Cantilevers in Varying} Humidity Conditions. Figures 3 and 4 show the resonance frequency response of cantilevers, from array types A and B, to changes in humidity, respectively. Agarose-functionalised cantilevers show an increased response to changes in humidity. This is due to an increase in mass on the cantilever surface caused by the absorption of water by the agarose layer. Increasing the incubation time in agarose solution during functionalisation results in a thicker layer and hence a greater response to humidity fluctuations (Figure 4). There is seen to be a correlation between frequency noise levels in the device and the incubation time of the cantilevers in the agarose solution during functionalisation. For incubation times of 1 , 5 , and 10 seconds, noise levels are found to be in the order of $4,5.5$, and $7 \mathrm{~Hz}$, respectively, at experimental conditions $\left(94 \% \mathrm{RH}, 30^{\circ} \mathrm{C}\right)$ (data not shown). The mass responsivity 


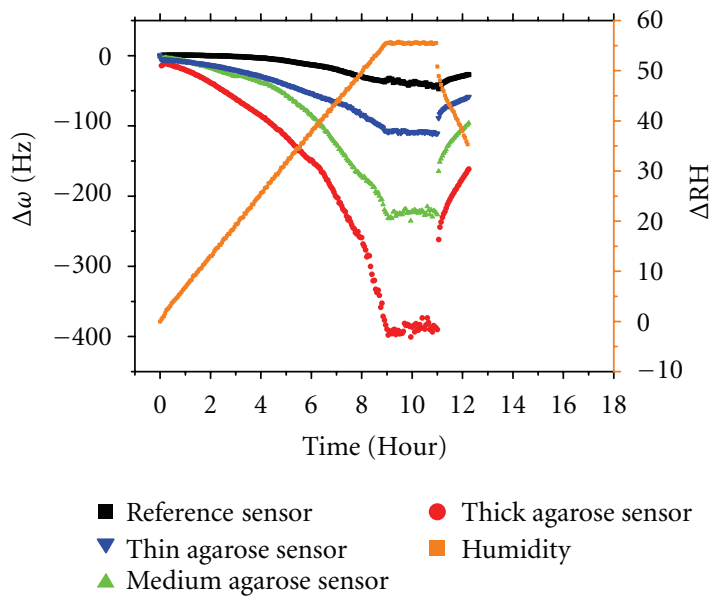

FIGURE 4: Resonance frequency response of agarose-functionalised cantilevers. The thin, medium and thick agarose sensors correspond to 1-, 5-, and 10-second incubation times during functionalisation, respectively. The response to humidity changes increases with increasing layer thickness. Frequency noise levels are also seen to increase slightly with increasing layer thickness. Zero point on right hand axis corresponds to $36 \% \mathrm{RH}$.

of our sensor when operated in its first resonance mode is $\sim 51 \pm 1 \mathrm{pg} / \mathrm{Hz}$. It should be noted that the minimal detectable mass increases with the thickness of agarose on the cantilever surface due to increased noise levels.

3.2. Aspergillus niger Growth Detection. Figure 5 shows resonance frequency shift $\left(\Delta \omega=\omega_{0}-\omega_{1}\right)$ versus time for two A. niger spore-coated cantilevers. The observed shift is as a result of spores starting to germinate and spread mycelium along the cantilever. To put this into context, the mass of an individual spore (diameter $\sim 4 \mu \mathrm{m}$ ) is $\sim 34 \mathrm{pg}$, which increases to $\sim 270 \mathrm{pg}$ due to swelling before germination (diameter $\sim 8 \mu \mathrm{m}$ ). This value is well within our detection limits indicated earlier. The growing mycelium assimilates nutrients and mostly water from the nutritive layer, which then absorbs water to remain in equilibrium with its surroundings. The detection of vital fungal growth is possible in less than $4 \mathrm{~h}$. This value is in agreement with that reported in $[13,14]$. The use of our fibre optic detection technique allows data to be recorded continuously. The use of an automated system allows experiments to be run over longer periods of time $(>48 \mathrm{~h}$ ) which provides further insight into the dynamics of mycelium growth. Approximately $28 \mathrm{~h}$ after the start of the experiment, an increase in resonance frequency, and hence most likely a rise in stiffness $(k)$, was observed. We believe this increase to be as a result of the reinforcement of the cantilever by the growth of mycelium along its length (Figure 6). The effect of adsorbed mass stiffness and its position along the cantilever have been reported in $[17,18]$. These effects may apply here; however, further work is required to clarify this as the online imaging of spore growth would be required. We believe the jumps in resonance frequency observed at $\sim 33$ and $42 \mathrm{~h}$ for the cantilever with the denser spore coating (Figure 6(A); black curve) could be interpreted as a temporary buckling of the

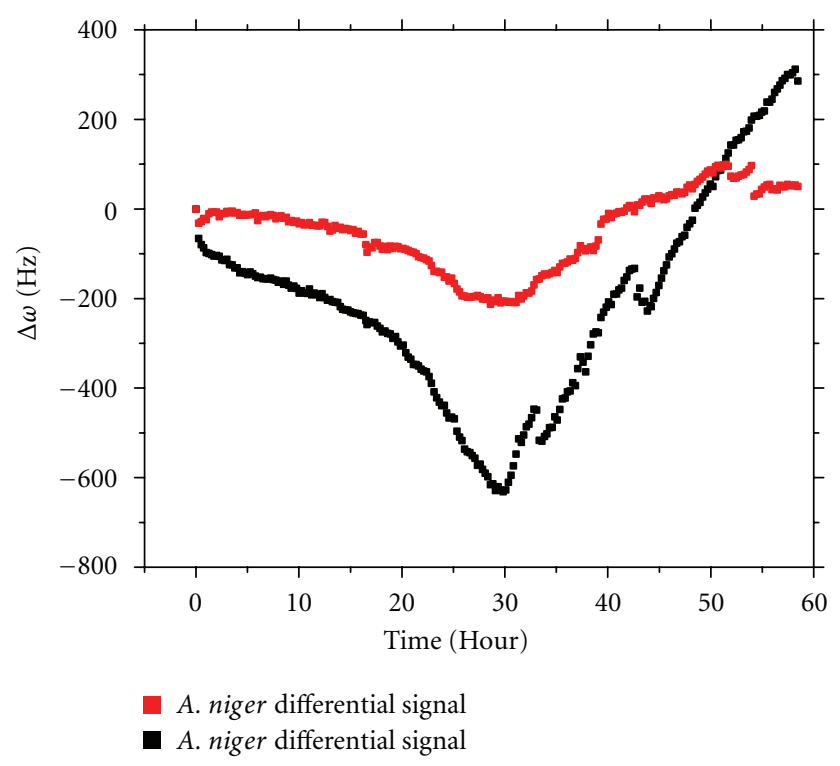

FIGURE 5: Differential nanomechanical response of $A$. niger functionalised cantilevers. The black and red curves correspond to Figure 6 (A and B, resp.). The agarose reference sensor remained stable with a standard deviation of $\sim 15 \mathrm{~Hz}$. Vital spore growth, which is manifested by a resonance frequency shift, is detected in less than $4 \mathrm{~h}$. An increase in resonance frequency is also observed after $\sim 28 \mathrm{~h}$. This is due to reinforcement of cantilevers caused by the growth of mycelium along their lengths. Different numbers of viable spores on each cantilever result in different magnitudes of response; however, growth rates are the same.

strained mycelium. Different magnitudes of response can be linked to a higher number of viable spores on one cantilever than the other as can be seen in Figure 6. One point to note is that the rate of growth is the same on both cantilevers as the individual sensor response seems to scale with the number of viable spores deposited.

\section{Conclusions}

A new home-built cantilever array device for fast microorganism growth detection has been successfully set up. Measurements involving growth of microorganisms require high humidity and elevated temperatures that are regulated with high precision. A fibre optic detection scheme was chosen to prevent adverse humidity effects to electronic parts which would have to be placed close by the cantilever structure under these environmental conditions. The nanomechanical change, induced by the viable growth of spores on cantilever sensors, can be continuously tracked. We demonstrated that the use of fibre optic beam injection and fibre optic beam modulation readout can be used to track the resonance frequency of cantilevers in microcantilever arrays over an extended period of time under high humidity and temperature. The resonance frequency of agarose-functionalised cantilevers in varying humidity conditions was instantaneously tracked revealing a mass responsivity for the thin agarosecoated sensors of $\sim 51 \pm 1 \mathrm{pg} / \mathrm{Hz}$. Frequency noise levels were also found to have a dependence on the thickness of the 


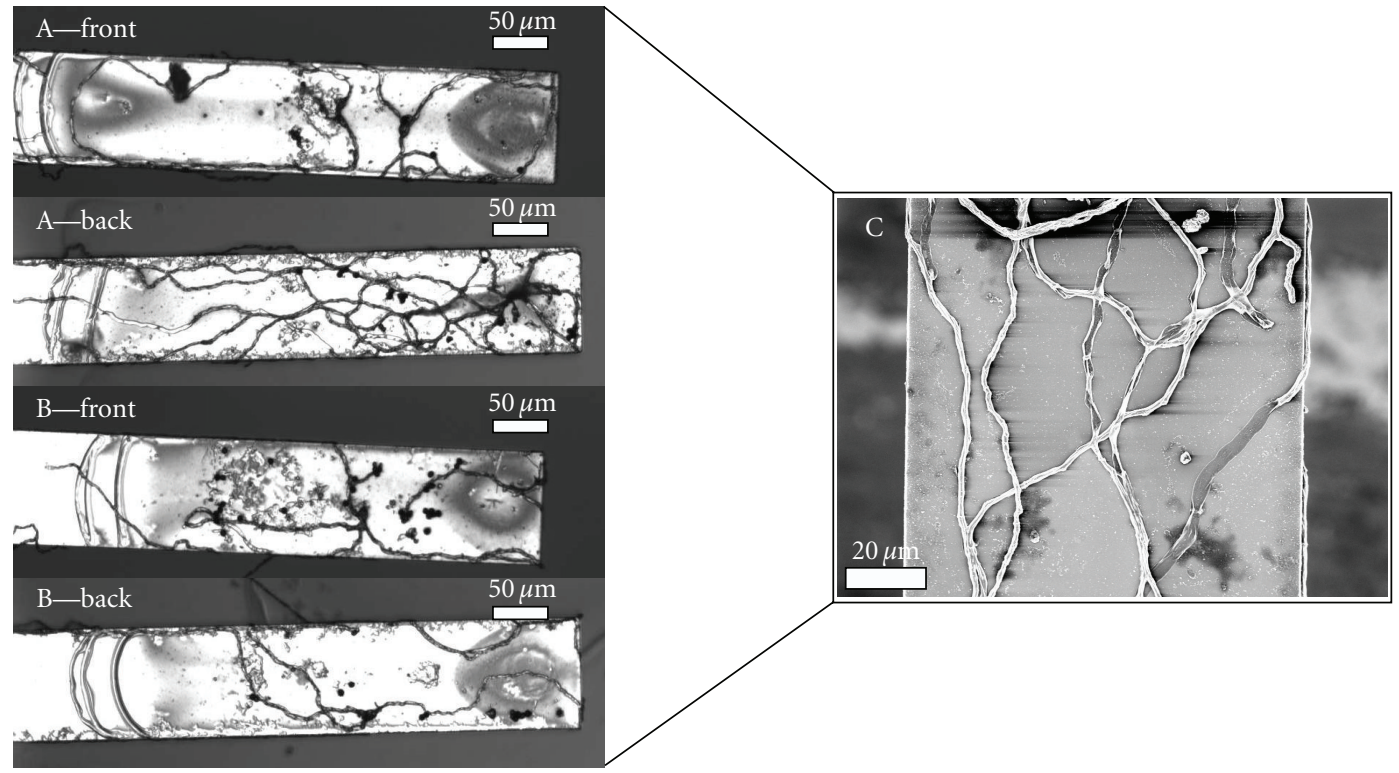

FIGURE 6: Optical microscope images of the front and backside of two A. niger coated cantilevers. Figure 6 (A and B) show the front and backsides of cantilevers which correspond to the black and red curves from Figure 5, respectively. Figure C shows an SEM image of mycelium on the surface of a cantilever. Mycelium thicknesses $(\sim 2 \mu \mathrm{m})$ are of the same order of magnitude when compared to the cantilever thickness $(7 \mu \mathrm{m})$. The growth of mycelium proceeds at a rate of $\sim 9 \mu \mathrm{m} / \mathrm{h}$ along the length of cantilevers.

agarose layer favouring thin layers produced during 1 second functionalisations. The detection of growth of viable $A$. niger spores was recorded in less than $4 \mathrm{~h}$. The use of an automated integrated detection system allowed nanomechanical data to be recorded continuously and growth of viable species to be monitored for $>48 \mathrm{~h}$, revealing additional insight into the dynamics of mycelium growth. We postulate that extended growth of micron-sized mycelium along the longitudinal axis of micron-sized cantilevers is inducing a reinforcement of the nanomechanical properties of the micron thin cantilever structures. It was observed that micromycelia are able to grow with a speed of up to $\sim 9 \mu \mathrm{m} / \mathrm{h}$ under optimal growth conditions and therefore have comparable dimensions to the sensors on which they are grown. The fast detection of viable microorganism, as demonstrated in this paper, is important for routine quality control tests which are required to ensure that microbial contamination does not occur and that preservatives are functional.

\section{Acknowledgments}

This work was supported by Science Foundation Ireland under the CSET Scheme (SFI08/CE/I1432), PI Scheme (SFI/09IN/1B2623), and Novartis Science Foundation. The authors would also like to acknowledge the use of highresolution imaging equipment located in the Advanced Microscopy Laboratory (AML, Trinity Technology and Enterprise Campus, Dublin 2, Ireland).

\section{References}

[1] EuropeanPharmacopoeia5.0, "Efficacy of Antimicrobial Preservation," 2005.
[2] N. Backmann, C. Zahnd, F. Huber et al., "A label-free immunosensor array using single-chain antibody fragments," Proceedings of the National Academy of Sciences of the United States of America, vol. 102, no. 41, pp. 14587-14592, 2005.

[3] T. Braun, N. Backmann, M. Vögtli et al., "Conformational change of bacteriorhodopsin quantitatively monitored by microcantilever sensors," Biophysical Journal, vol. 90, no. 8, pp. 2970-2977, 2006.

[4] J. Zhang, H. P. Lang, F. Huber et al., "Rapid and label-free nanomechanical detection of biomarker transcripts in human RNA," Nature Nanotechnology, vol. 1, no. 3, pp. 214-220, 2006.

[5] J. Zhang and H. F. Ji, "An Anti E. Coli O157:H7 antibodyimmobilized microcantilever for the detection of Escherichia coli (E. coli)," Analytical Sciences, vol. 20, no. 4, pp. 585-587, 2004.

[6] M. Li, H. X. Tang, and M. L. Roukes, "Ultra-sensitive NEMSbased cantilevers for sensing, scanned probe and very highfrequency applications," Nature Nanotechnology, vol. 2, no. 2, pp. 114-120, 2007.

[7] B. Ilic, D. Czaplewski, M. Zalalutdinov et al., "Single cell detection with micromechanical oscillators," Journal of Vacuum Science and Technology B, vol. 19, no. 6, pp. 2825-2828, 2001.

[8] A. Gupta, D. Akin, and R. Bashir, "Single virus particle mass detection using microresonators with nanoscale thickness," Applied Physics Letters, vol. 84, no. 11, pp. 1976-1978, 2004.

[9] T. Braun, M. K. Ghatkesar, N. Backmann et al., "Quantitative time-resolved measurement of membrane protein-ligand interactions using microcantilever array sensors," Nature Nanotechnology, vol. 4, no. 3, pp. 179-185, 2009.

[10] A. K. Naik, M. S. Hanay, W. K. Hiebert, X. L. Feng, and M. L. Roukes, "Towards single-molecule nanomechanical mass spectrometry," Nature Nanotechnology, vol. 4, no. 7, pp. 445450, 2009.

[11] K. Y. Gfeller, N. Nugaeva, and M. Hegner, "Rapid biosensor for detection of antibiotic-selective growth of Escherichia coli," 
Applied and Environmental Microbiology, vol. 71, no. 5, pp. 2626-2631, 2005.

[12] K. Y. Gfeller, N. Nugaeva, and M. Hegner, "Micromechanical oscillators as rapid biosensor for the detection of active growth of Escherichia coli," Biosensors and Bioelectronics, vol. 21, no. 3, pp. 528-533, 2005.

[13] N. Nugaeva, K. Y. Gfeller, N. Backmann et al., "An antibodysensitized microfabricated cantilever for the growth detection of Aspergillus niger spores," Microscopy and Microanalysis, vol. 13, no. 1, pp. 13-17, 2007.

[14] N. Nugaeva, K. Y. Gfeller, N. Backmann, H. P. Lang, M. Düggelin, and M. Hegner, "Micromechanical cantilever array sensors for selective fungal immobilization and fast growth detection," Biosensors and Bioelectronics, vol. 21, no. 6, pp. 849-856, 2005.

[15] G. Meyer and N. M. Amer, "Novel optical approach to atomic force microscopy," Applied Physics Letters, vol. 53, no. 12, pp. 1045-1047, 1988.

[16] A. Bietsch, J. Zhang, M. Hegner, H. P. Lang, and C. Gerber, "Rapid functionalization of cantilever array sensors by inkjet printing," Nanotechnology, vol. 15, no. 8, pp. 873-880, 2004.

[17] J. Tamayo, D. Ramos, J. Mertens, and M. Calleja, "Effect of the adsorbate stiffness on the resonance response of microcantilever sensors," Applied Physics Letters, vol. 89, no. 22, Article ID 224104, 2006.

[18] D. Ramos, J. Tamayo, J. Mertens, M. Calleja, and A. Zaballos, "Origin of the response of nanomechanical resonators to bacteria adsorption," Journal of Applied Physics, vol. 100, no. 10, Article ID 106105, 2006. 

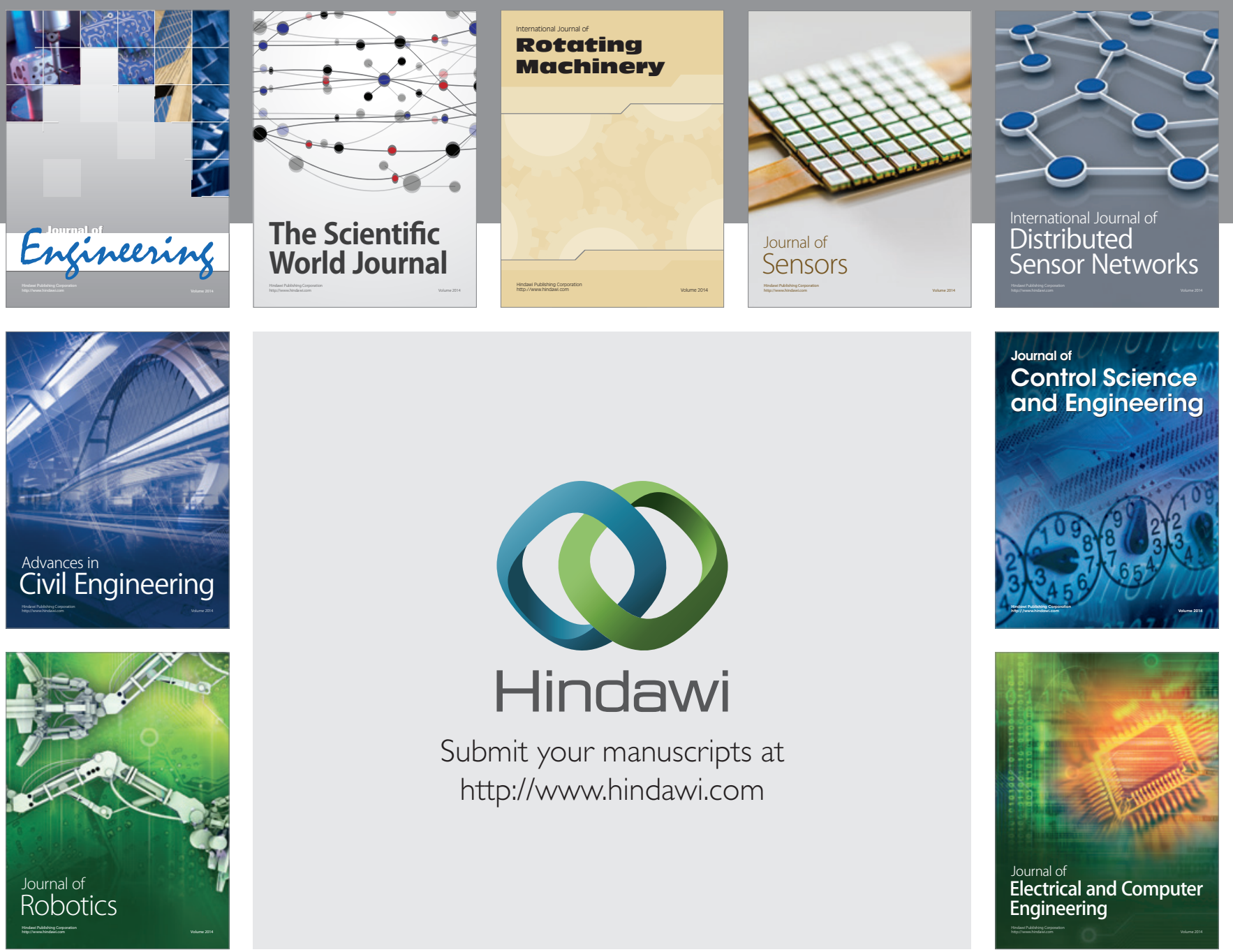

Submit your manuscripts at

http://www.hindawi.com
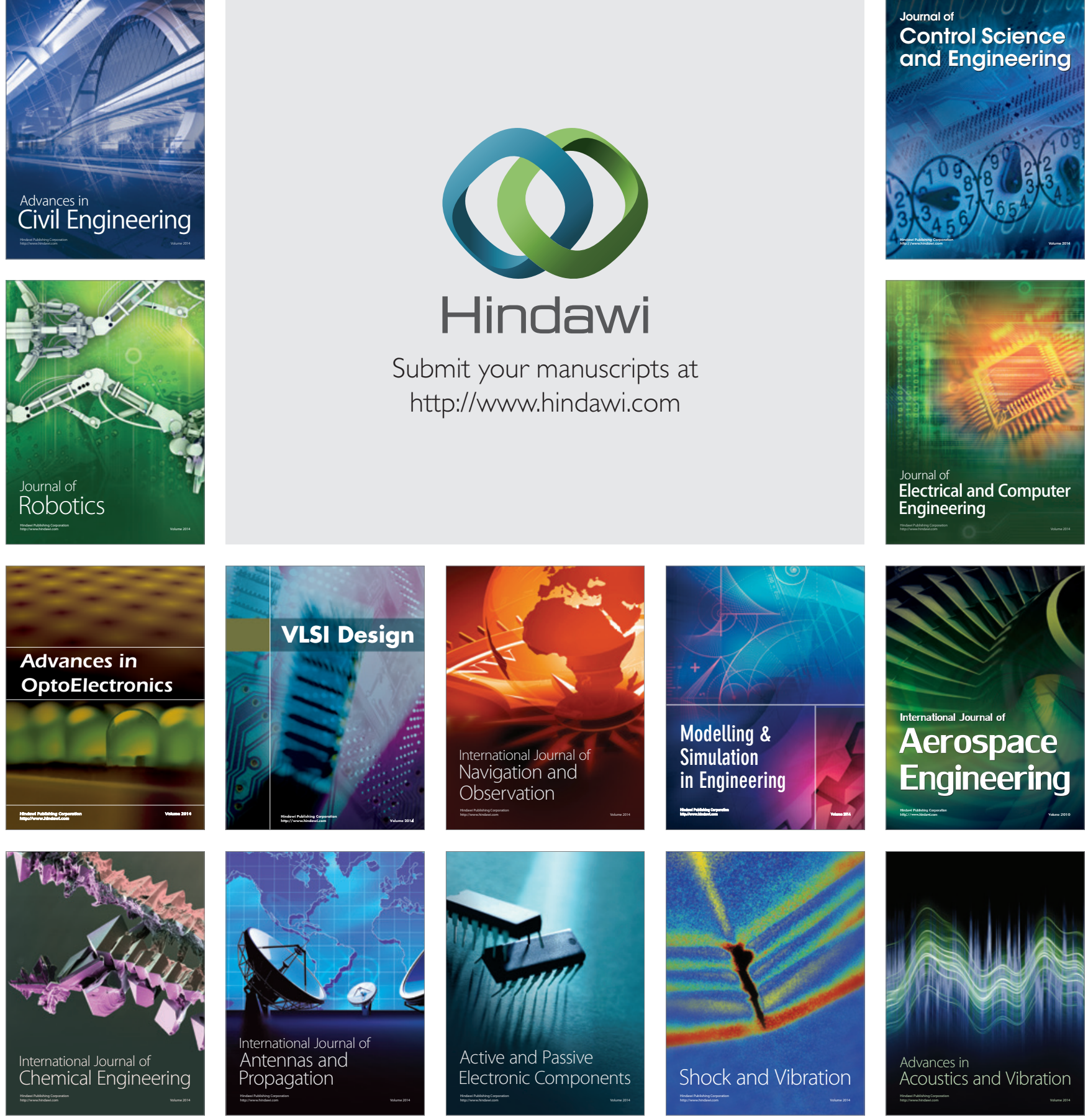